\title{
Exogenous sex hormone effects on brain microstructure in women: a diffusion MRI study in the UK Biobank
}

\author{
Leila Nabulsi ${ }^{\mathrm{a}}$, Katherine E. Lawrence ${ }^{\mathrm{a}}$, Vigneshwaran Santhalingam ${ }^{\mathrm{a}}$, Zvart Abaryan ${ }^{\mathrm{a}}$, Christina P. \\ Boylea, Julio E. Villalon-Reina a, Talia M. Nira, Iyad Ba Garia, Alyssa H. Zhua, Elizabeth Haddad ${ }^{\text {a }}$, \\ Alexandra M. Muira, Neda Jahanshad ${ }^{\mathrm{a}}$, and Paul M. Thompson ${ }^{\mathrm{a}}$ \\ ${ }^{a}$ Imaging Genetics Center, Mark and Mary Stevens Neuroimaging \& Informatics Institute, \\ University of Southern California, Marina del Rey, CA 90292, USA
}

\begin{abstract}
This study used advanced diffusion-weighted MRI (dMRI) to examine the association between exogenous sex-hormone exposure and the brain's white matter aging trajectories in a large population-based sample of women. To investigate the effect of pre- and post-menopausal sex hormones on brain aging, cross-sectional brain dMRI data from the UK Biobank was analyzed using 3 diffusion models: conventional diffusion tensor imaging (DTI), the tensor distribution function (TDF), and neurite orientation dispersion and density imaging (NODDI). Mean skeletonized diffusivity measures were extracted and averaged across the whole brain, including fractional anisotropy, isotropic volume fraction, intracellular volume fraction and orientation dispersion index. We used general linear models and fractional polynomial regressions to characterize age-related trajectories in white matter measures following hormone therapy (HT) and oral contraceptive (OC) use in women (HT analysis: $\mathrm{N}=8,301$; OC analysis: $\mathrm{N}=8,913$ ). Sex hormone treatment (HT and OC) was statistically associated with the aging trends in white matter measures. Estrogen therapy alone appeared to exert a neuroprotective effect on age-related white matter processes, compared to HT containing both estrogen and progestin therapy - which was associated with accelerated aging-related processes in women. These results support the hypothesis that exogenous sex hormone exposure may impact white matter aging; white matter metrics may also be sensitive to sex hormone levels in women. Furthermore, we discuss the necessity to test alternative models for lifespan trajectories beyond popular linear and quadratic models, especially when dealing with large samples. Fractional polynomial models may provide a more adaptive alternative to linear or quadratic models.
\end{abstract}

Keywords: diffusion magnetic resonance imaging (dMRI), aging, sex hormones, hormone therapy, DTI, TDF, NODDI.

\section{INTRODUCTION}

Accurate characterization of how and where the brain's white matter microstructure changes with age is a vital goal in brain aging research. Late-life cognitive decline may be caused, in part, by microstructural deteriorations in the brain's neural pathways. Processes such as neuronal and glial cell loss, impaired myelin production, and axonal demyelination, may impair information transfer efficiency in the brain's white matter networks. The white matter disconnection theory is largely supported by emerging data from diffusion MRI (dMRI) studies of the brain. Diffusion MRI is an in vivo imaging technique that allows non-invasive, quantitative investigation of microstructural differences in white matter tracts by exploiting the Brownian motion of water molecules, and the dependency of this diffusion process on the cellular environment ${ }^{1}$. Studying brain changes during development and aging is key to understanding processes that govern brain maturation and decline, and may help to identify mechanisms of neurodevelopmental and age-related chronic disorders. Also, statistical data on normal aging in populations of healthy individuals can be used to identify imaging markers for early detection and to define people at greater risk of developing age-related neurodegenerative disorders such as Alzheimer's disease (AD).

In women, higher levels of sex-related hormones (such as estrogen) may be associated with increased risk for age-related neurodegenerative diseases such as $\mathrm{AD}^{2}$. On average, women above 40 years of age are at a greater risk of developing $\mathrm{AD}$ than age-matched men. Endogenous and exogenous estrogen levels also influence this risk ${ }^{3,4}$. Still, we know very little 
about how sex hormone levels affect brain aging. Changes in sex hormones such as estradiol may alter brain plasticity ${ }^{5,6,7}$. Several brain structural changes have been associated with estrogen fluctuations in pregnant women ${ }^{8}$ and in premenopausal women across the menstrual cycle ${ }^{9}$. Apparently beneficial effects of endogenous estrogen levels have been reported for gray matter, with larger brain volumes for women during the reproductive years ${ }^{10,11}$. Even so, negative effects of endogenous estrogen levels on brain regional volumes have been reported in menopausal women ${ }^{12}$.

Hormone therapy (HT) and oral contraceptives (OC) are both common sources of exogenous estrogen in women's menopausal and reproductive years, respectively. The first study to report negative effects of exogenous estrogen levels in women was the Women's Health Initiative Memory Study (WHIMS) ${ }^{12}$, describing greater brain atrophy in women $\geq 65$ years treated with conjugated estrogen, both alone and in combination with progestin. More evidence arose in a longitudinal study that reported increasing ventricular enlargement over 4 years in recently menopausal women ${ }^{13}$. However, other neuroimaging studies suggest a neuroprotective effect of HT on gray matter ${ }^{14}$, white matter and ventricular volumes ${ }^{15}$.

A number of clinical studies of HT exposure effects on the brain led to the critical period hypothesis which states that HT may be neuroprotective if initiated near the time of cessation of ovarian function - that is, within around 5 years of menopause ${ }^{16,17}$. While most evidence suggests that estrogen exerts a neuroprotective effect in pre-menopausal women, whether post-menopausal estrogen exposure is beneficial or detrimental for cognition is still an open question. A large study (N=4,894, WHIMS) reported for the first time that post-menopausal HT exposure may be detrimental for cognition and may increase AD risk in women ${ }^{4}$. However, subsequent studies using the WHIMS data ${ }^{18}$ and other studies such as KEEPS $^{19}$ and ELITE ${ }^{20}$ indicated that women prescribed HT much later (>10 years) after menopause may be at higher risk of $\mathrm{AD}$; this risk was perhaps mediated by the impact of Type 2 diabetes mellitus. Thus, the duration of HT and age of onset may be key factors in estrogen-mediated effects on the brain.

Moreover, despite the increasing use of OCs globally and within the United States ${ }^{21}$, exogenous estrogen exposure via OCs is understudied, and their impact on white matter is unclear (structural and functional findings are reviewed in ${ }^{22}$ ). The OC literature has focused mainly on comparing cognitive performance in users and non-users across the menstrual cycle of pre-menopausal women ${ }^{23,24}$, but these studies are inconclusive due to the small sample sizes considered.

Determining effects on the brain's white matter of HT in pre- and post-menopausal years would require decades of longitudinal research following individuals across the lifespan. However, non-invasive in vivo imaging biomarkers that are associated with age and with cognition have been increasingly used as proxies to assess effects of menopausal HT on the brain (with large recent studies in the UK Biobank ${ }^{25,26}$ ). In this current paper, we set out to measure and model ageassociated effects on white matter microstructure to infer possible effects of exogenous sex hormones on the brains of preand post-menopausal women. We primarily sought to examine the association between exogenous sex hormone exposure and aging white matter trajectories in women using HT or OC, compared to non-users. Drawing on the existing literature on sex hormone effects on the brain, we hypothesized that sex hormone therapy - HT or OC - would negatively impact women's white matter aging. Post-hoc exploratory analyses were carried out to account for differences in HT formulation (estrogen or estrogen + progestin). Supplemental analyses were carried out to test the link between duration of therapy, age at HT/OC onset and white matter aging on diffusivity metrics in women. We analyzed single- and multi-shell diffusionweighted brain MRI data from the UK Biobank ${ }^{26}$ and processed them using 3 increasingly complex diffusion models. Diffusion indices were extracted and averaged across a whole brain white matter skeleton. We used general linear models and fractional polynomial (FP) regression to characterize sex hormone effects on age-related trajectories in white matter. Finally, we computed normalized centile curves to visualize white matter aging trajectories for the major diffusivity metrics. 


\section{MATERIALS AND METHODS}

\subsection{Subjects and Image Acquisition}

\subsubsection{Sample}

Our sample was drawn from the UK Biobank ${ }^{27}$ and included N=8,301 (HT analysis) and N=8,913 (OC analysis) premenopausal and post-menopausal women aged 45-80 years old. The UK Biobank is currently the largest prospective study of aging, gathering extensive questionnaires, physical and cognitive measures and biological samples in a populationbased cohort of middle- to older-aged adults living in the United Kingdom (UK). Here we analyzed diffusion-weighted magnetic resonance imaging (dMRI) data (Application ID \#11559) to elucidate pre- and post-menopausal exogenous hormone effects in women. We examined age effects on in WM measures derived from both standard and advanced dMRI modeling techniques (DTI, TDF, NODDI; defined below).

\subsubsection{MRI processing}

For a more complete overview of the UKB data acquisition, neuroimaging protocol, and validation, see work by Miller ${ }^{27}$ and Alfaro-Almagro ${ }^{28}$. In brief, diffusion-weighted images were acquired at $b=1000$ and $2000 \mathrm{~s} / \mathrm{mm}^{2}$ along with 50 noncoplanar diffusion directions per diffusion shell with $5 b=0 \mathrm{~s} / \mathrm{mm}^{2}$ (and $3 b=0$ blip-reversed) using a standard ('monopolar') Stejskal-Tanner pulse sequence (voxel dimension: $(2 \mathrm{~mm})^{3}$ isotropic, field of view: 104x104x72 mm; gradient timings: small delta $=21.4 \mathrm{~ms}$, big delta $=45.5 \mathrm{~ms}$ ) for a total imaging time of 7 minutes.

Diffusion-weighted images were processed using a harmonized processing pipeline. All images underwent pre-processing, involving noise correction ${ }^{29,30}$, Gibbs-ringing correction ${ }^{31}$, estimation of echo-planar imaging distortions, motion and eddy current and susceptibility distortion corrections ${ }^{32,33}$, spatial smoothing ( $f_{\text {slmaths }}$ in FSL) with Gaussian kernel (1 mm FWHM) $)^{3}$ and diffusion metric estimation.

Diffusion tensor imaging (DTI) fitting was performed on the $b=1000 \mathrm{~s} / \mathrm{mm}^{2}$ shell (50 directions) using the DTI fitting (DTIFIT) tool to create maps of tensor-derived fractional anisotropy (FA), representing the degree of anisotropic diffusivity - often considered to measure the degree of directional coherence within a fiber bundle ${ }^{34,35}$.

Diffusion MRI data ( $b=1000 \mathrm{~s} / \mathrm{mm}^{2}, 50$ directions) was also used as input data to estimate the tensor distribution function (TDF) at each voxel in the image ${ }^{36}$. This approach extends multi-tensor models of diffusion to describe intravoxel fibers mathematically as a probabilistic collection of tensors, or, alternatively, a continuous mixture of Gaussian densities. The fitted TDF function was employed to create diffusion maps of fractional anisotropy, based on the mixture of tensors. This approach overcomes some known limitations of traditional diffusion tensor models in regions of fiber mixing or crossing ${ }^{37,38}$.

In addition to TDF and DTI models, the dMRI data ( $b=1000$ and $2000 \mathrm{~s} / \mathrm{mm}^{2}, 100$ directions) were input into Neurite Orientation Dispersion and Density Imaging (NODDI) ${ }^{39}$ models, using the $\mathrm{AMICO}^{40}$ (Accelerated Microstructure Imaging via Convex Optimization) tool, to obtain the following voxel-wise microstructural parameters: ODI (orientation dispersion index, a measure of within-voxel tract white matter disorganization), ICVF (intracellular volume fraction, and index of white matter neurite density) and ISOVF (isotropic or free water volume fraction).

Voxel-wise statistical analysis of the images was performed using Tract-based Spatial Statistics (TBSS ${ }^{41}$; Figure 1) using a standardized processing pipeline (https://enigma.ini.usc.edu/wpcontent/uploads/DTI_Protocols/ENIGMA_TBSS_protocol_USC.pdf), with the following steps. After generating the FA maps from DTI and TDF measures respectively, images were aligned onto a standard-space white matter skeleton (ENIGMA white matter template, representing the center of all white matter FA voxels in MNI space) using ANTs registration. The aligned FA maps were then averaged to produce a group mean image, which was used to generate an FA skeleton highlighting the tracts common to the entire group. For each subject, an FA threshold of 0.2 was used before projecting the aligned FA map onto this skeleton. In addition to FA, diffusivity maps based on measures of ODI, ICVF, 
and ISOVF were also generated using the same steps outlined above. The Johns Hopkins University (JHU) WM tractography atlas in FSL was used as a standard for WM parcellation. WM was parcellated into 48 regions of interest (ROIs) using the 1-mm JHU-ICBM labels ${ }^{42,43}$. Average whole-brain measures of DTI FA, TDF FA, NODDI ODI, ISOVF, and ICVF were extracted for each participant. Qualitative quality control was carried out at each step of the TBSS pipeline, to ensure accuracy of each step, with a specific focus on the alignment of each individual's FA, ODI, ISOVF, and ICVF maps to the mean FA skeleton averaged across the group.

\subsubsection{Analyses}

\subsubsection{Statistical Approaches}

To statistically estimate effects of exogenous sex hormone exposure on aging trajectories, values from each respective diffusion metric (DTI FA, TDF FA, NODDI ODI, ISOVF and ICVF) were fitted as a dependent variable in general linear models, with mean-centered age and age $^{2}$ as independent variables, using R version 3.6.1 (R Core Team, Vienna, Austria). Main analyses included interactions between age and HT/OC status. Follow-up exploratory analyses included separate interactions between age and HT type, duration of HT/OC, and age at HT/OC onset. Note that the hormone exposure and hormone treatment are not randomized across individuals, as would be the case in a randomized clinical trial; instead, the study is a naturalistic epidemiological study of a large population (for assumptions and limitations of this approach, see Discussion). Classic multiple regression models assume a linear relationship between independent and dependent variables. However, this assumption might be questioned for age effects on the brain, which are the aggregate of a vast number of cellular processes, each with potentially different profiles of acceleration and decline. As the age effects are likely highly nonlinear, with a functional form that is not known a priori, fractional polynomials may offer a more adaptive modeling approach ${ }^{43}$. Polynomial regressions are extensions of linear regressions, but they may also be considered as a special case of multiple linear regression, that includes both power and logarithmic terms. We modeled the effect of age on diffusion metrics using higher order polynomial (FP) regression ${ }^{44,45}$. Specifically, we used the multivariate fractional polynomial (MFPI) package implemented in STATA software version 16.1 (Stata Corp., College Station, TX). The MFPI package used a predefined set of power terms $(-2,-1,-0.5,0.5,1,2,3)$ and the natural logarithm function, and up to two power combinations to identify the best fitting model. (Findings from MFP relative to traditional regression models are discussed later). Furthermore, centile curves ${ }^{46,47,48}$ for diffusion metrics with respect to age were calculated and plotted for each metric (Phyton); kernel density plots, indicating the degree of data point overlap (and sampling density across the age range), are included in the plots.

\subsubsection{Clinical variables}

Exogenous exposure was estimated by using HT and OC variables. For HT analyses, 3,106 users and 5,195 never-users were included in the analyses. For OC analyses, 7,136 users and 1,177 never-users were included. Women who had never used HT or OC were coded as 0 , while current and former users were coded as 1 . Multiple regression analyses were run to investigate the relationship between estimates of exogenous hormone exposure and the brain's white matter aging patterns. As differences in HT compound composition (combined or unopposed estrogen treatment) may have a differential impact on aging trajectories ${ }^{3,19}$, we carried out exploratory post-hoc investigations on aging trajectories for estrogen onlytherapy versus combination (estrogen and progestin) therapy. For this question, data was available for only a subset of the UK Biobank participants in the sample we studied (estrogen only, $n=306$; combination, $n=102$ ).

For any of the variables of interest, women who had missing data, responded 'do not know' or 'preferred not to answer' or missing data were excluded from each analysis. Main nuisance covariates included years of education, waist/hip ratio, population structure, measured using the first four genetic principal components obtained from the UK Biobank's genetic ancestry analyses, and the Townsend index, a measure of socioeconomic status. In detail, educational attainment was assessed using a sociodemographic questionnaire in which participants reported their completed qualification level (UK education system). These results were generalized beyond the UK education system and converted to the corresponding number of years using ISCED harmonization guidelines ${ }^{49}$ and dichotomized into 'college' ( $>=17$ years) versus 'high school' education. Waist-to-hip ratio is a common index of central fat distribution ${ }^{50}$ and used as a proxy of atherosclerotic burden in overweight individuals and postmenopausal women ${ }^{51,52}$. Genetic principal components were regressed out to account for differences in ethnicity within the sample; only the first 4 components were considered as these have been 
previously shown to capture most of the population structure. Participants' socioeconomic status (SES) was measured using the Townsend Index ${ }^{53}$, a measure of societal deprivation based on the postal code of each participant - this index has been often used as a proxy measure of SES $^{54}$. To assess robustness of the findings, different sets of nuisance covariates were considered in the model. Consequently, all statistical analyses were repeated to include measures of arterial stiffness as a proxy for aortic blood pressure, and body mass index (BMI) instead of the waist-to-hip ratio (supplemental analyses). In subsequent analyses, we considered whether participants had a history of hysterectomy and bilateral oophorectomy, and also included the number of childbirths, as covariates (supplemental analyses). In addition, model-specific covariates were included to correct for potential confounds or factors that might influence the effects of covariates of interest; these included duration of HT/OC usage, the age at which participant began HT/OC therapy, and age at menopause (all mean centered for the interaction analyses).

\section{RESULTS}

\subsection{Exogenous sex hormone exposure}

\subsubsection{Age-related trajectories in HT}

Multiple linear regression including the main nuisance covariates showed no significant interaction between age and HT status on diffusion metrics (linear and quadratic $\mathrm{p}>0.05$ ). Supplemental analyses including BMI and arterial stiffness in the model showed an association between HT status and NODDI ODI (increased ODI, quadratic $p=0.04$; Figure 1) in never-users compared to users; including surgical history (hysterectomy and oophorectomy) and number of childbirths as nuisance covariates resulted in highly similar findings (quadratic, $\mathrm{p}=0.01$ ). Follow-up exploratory analyses examining combined or unopposed estrogen treatment showed an association between HT type and age on diffusion metrics DTI FA, TDF FA and increased NODDI ISOVF (post-hoc; range of linear $\mathrm{p}=0.008-0.03$; range of quadratic $\mathrm{p}=0.03-0.04$; Figure 2); findings were consistent across all nuisance covariate sets. Duration of HT use was associated with TDF FA and NODDI ISOVF in estrogen only compared to combination users (post-hoc, range of linear $\mathrm{p}=0.008-0.04$ ). Age at HT onset was associated with TDF FA and NODDI ISOVF (post-hoc, range of linear $\mathrm{p}=0.004-0.02$; Figure 3) in estrogen only compared to combination users; when adjusting for age at menopause, there was a significant association for NODDI ISOVF and age (post-hoc, linear $\mathrm{p}=0.007$ ). No significant association between age and duration of HT use or age at HT onset was seen for HT users compared to never-users on diffusion metrics.

MFPI testing did not replicate associations seen between age and HT status in linear regression models (across all nuisance covariates sets, $\mathrm{p}>0.05$ ); further, no significant association between age and duration of HT use or age at HT onset was observed using MFPI ( $p>0.05$ ). However, MFPI confirmed associations between age and HT type (estrogen alone and combination users) on diffusion metrics TDF FA (decreased, $\mathrm{p}<0.05$ ) and NODDI ISOVF (increased, $\mathrm{p}<0.05$ ).

\subsubsection{Age-related trajectories in $\mathrm{OC}$}

A linear regression including the main nuisance covariates showed a significant interaction between age and $\mathbf{O C}$ status on diffusion metrics DTI FA and TDF FA (both reduced; range of quadratic $\mathrm{p}=0.01-0.04$; Figure 4); supplemental analyses including other sets of covariates yielded similar findings. Follow-up exploratory analyses showed that duration of OC use and age at OC onset were significantly associated with reductions in DTI FA and TDF FA (post-hoc, duration: range of quadratic $\mathrm{p}=0.02-0.03$; onset: range of quadratic $\mathrm{p}=0.025-0.028$ ).

MFPI testing confirmed significant associations between age and DTI FA for OC status, duration and age of onset $(\mathrm{p}<0.05)$.

\subsubsection{Centile Curves of age-dependent trajectories}

Representative centile curves for each significant diffusion metric are presented in Figures 1, 2, and 4. Colored lines indicate 5th (black), 25th (blue), 50th (red), 75th (green), 95th (purple) centiles. Sampling density or distribution curves for age and each diffusion metric are shown at the top and right of each graph. Centile values were stratified by HT and 
bioRxiv preprint doi: https://doi.org/10.1101/2020.09.18.304154; this version posted September 20, 2020. The copyright holder for this preprint (which was not certified by peer review) is the author/funder, who has granted bioRxiv a license to display the preprint in perpetuity. It is made available under aCC-BY-ND 4.0 International license.

OC users/never-users and HT type (estrogen only/combination). Curves show age-dependent trajectories for all metrics; specifically, increased neurite dispersion (NODDI ODI), decreased fractional anisotropy (DTI-FA and TDF-FA), and increased free water (NODDI ISOVF) with age. More specifically, HT users showed on average, lower orientation dispersion (NODDI ODI) compared to never-users (Figure 1); estrogen users showed, on average, higher fractional anisotropy and lower free water values compared to estrogen and progestin users (Figure 2); finally, OC users showed higher fractional anisotropy (DTI-FA and TDF-FA) than never-users (Figure 4).
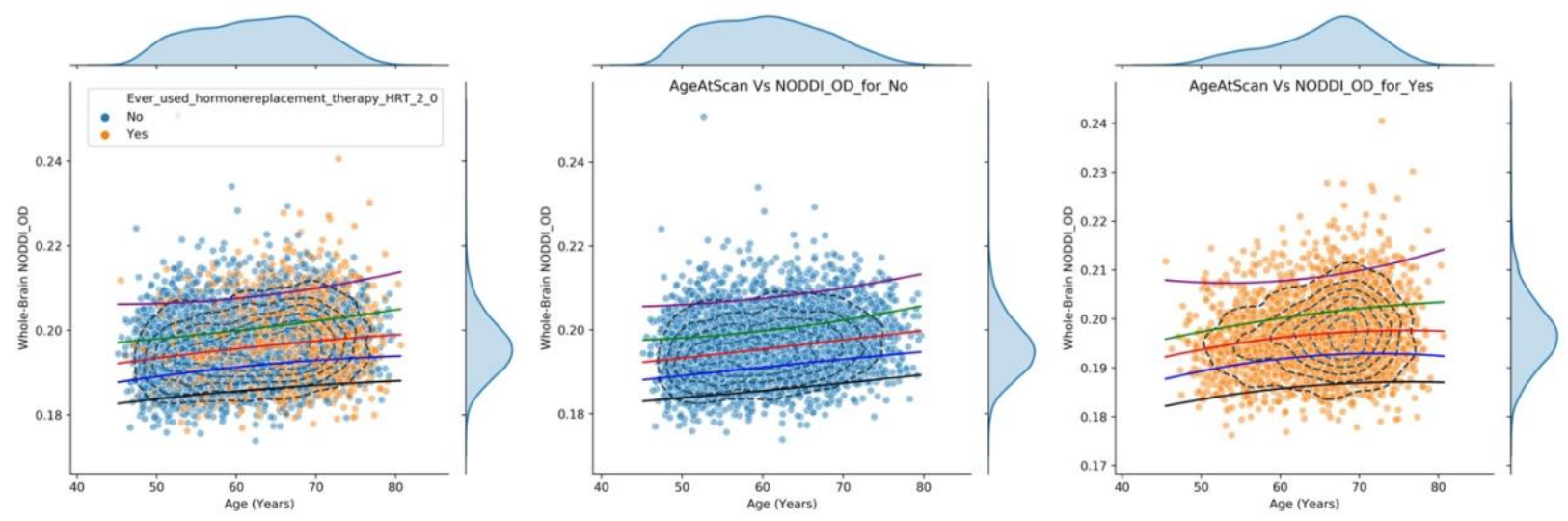

Figure 1. Whole brain averages for NODDI ODI shown for HT users compared to never-users, collectively (left panel) and separately by group (middle and right panels). HT users show lower NODDI ODI values compared to never-users. 
bioRxiv preprint doi: https://doi.org/10.1101/2020.09.18.304154; this version posted September 20, 2020. The copyright holder for this preprint (which was not certified by peer review) is the author/funder, who has granted bioRxiv a license to display the preprint in perpetuity. It is made available under aCC-BY-ND 4.0 International license.
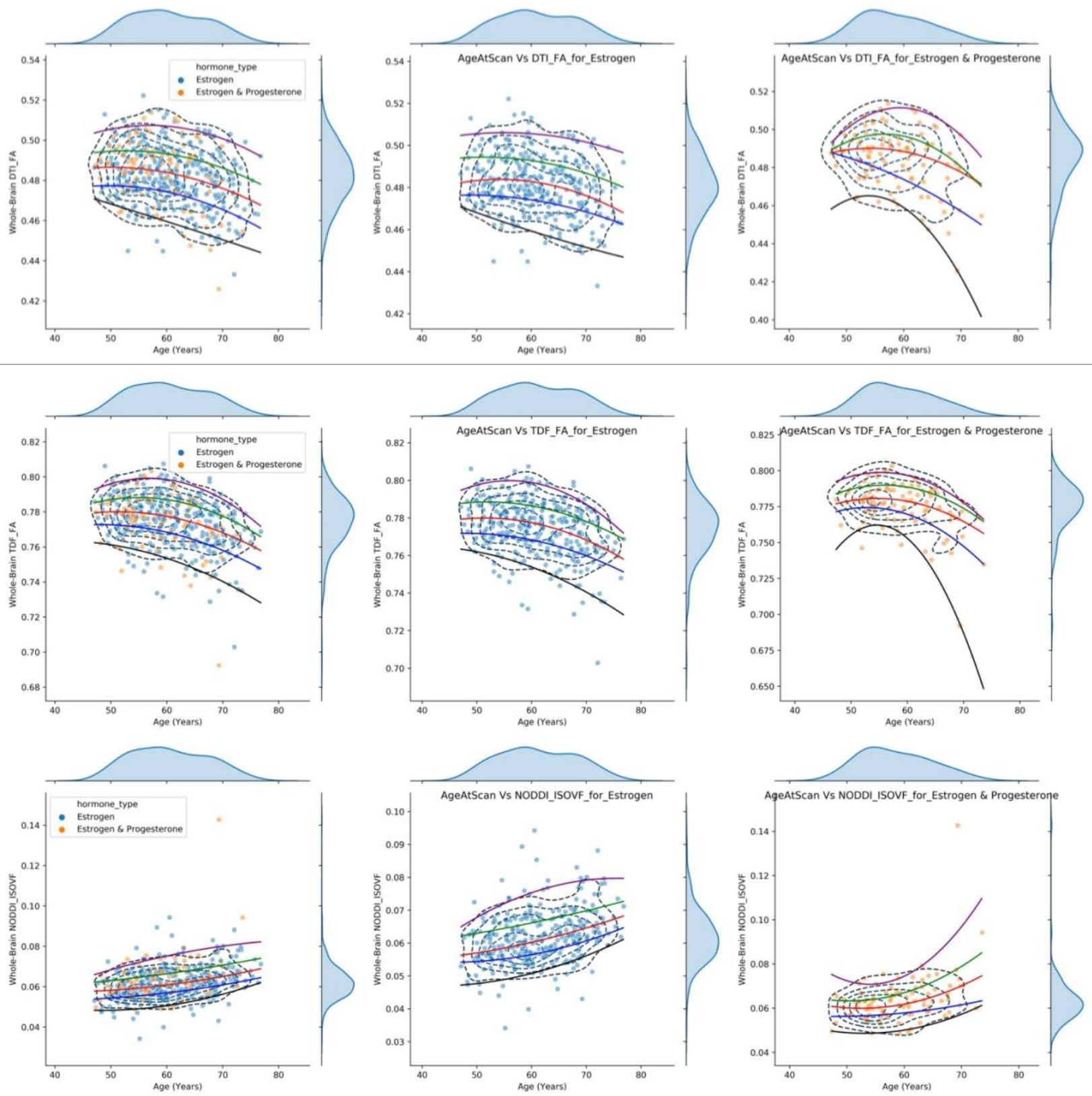

Figure 2. Whole brain averages for DTI-FA, TDF-FA and NODDI-ISOVF shown for estrogen users and combination users, collectively and separately by group. Estrogen only users show lower DTI-FA (top row) and TDF-FA (middle row), and higher NODDI-ISOVF (bottom row) compared to combination users. 
bioRxiv preprint doi: https://doi.org/10.1101/2020.09.18.304154; this version posted September 20, 2020. The copyright holder for this preprint (which was not certified by peer review) is the author/funder, who has granted bioRxiv a license to display the preprint in perpetuity. It is made available under aCC-BY-ND 4.0 International license.
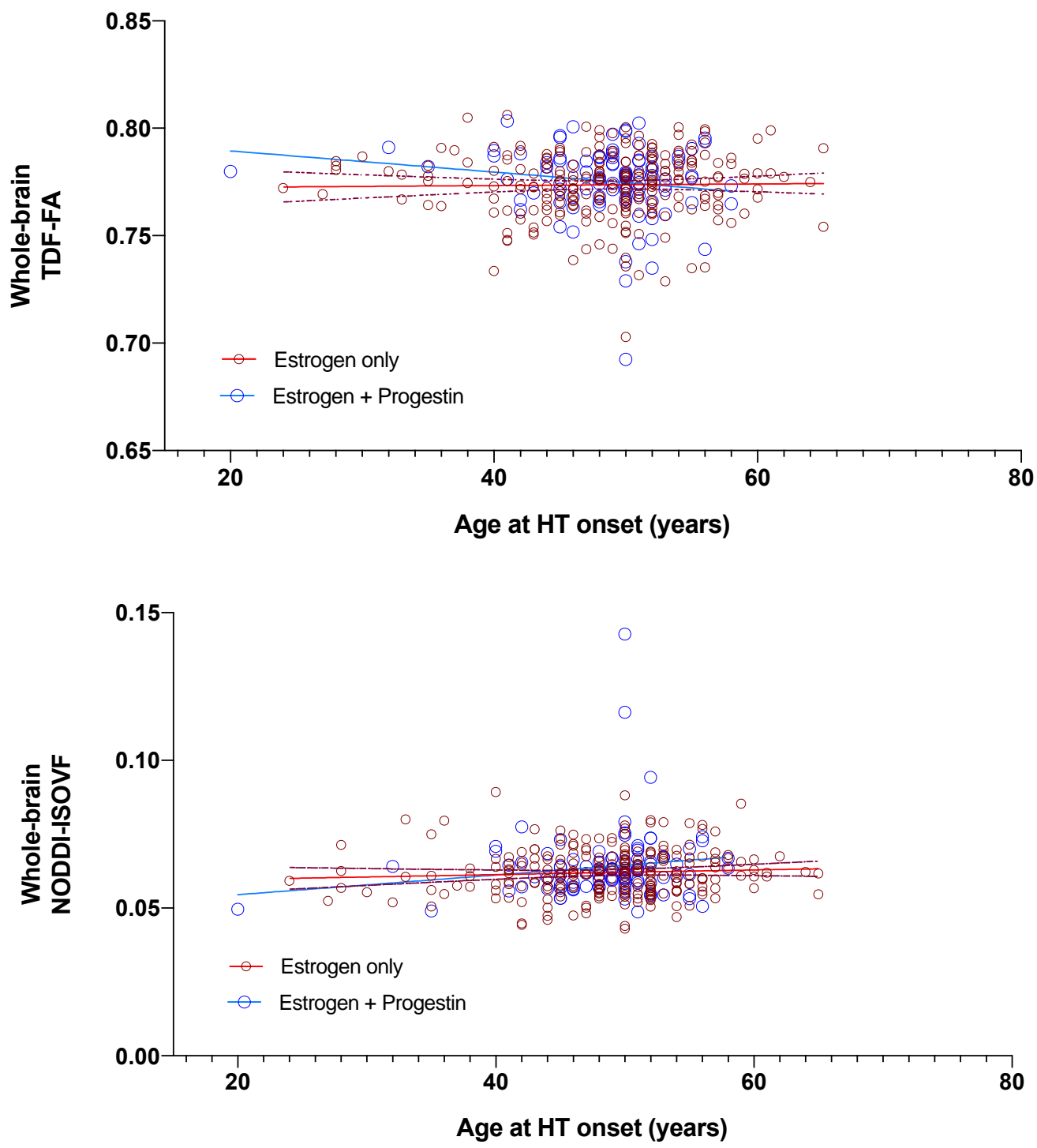

Figure 3. Later age at HT (estrogen and progestin) onset was associated with lower TDF FA (top) and increased NODDI ISOVF (bottom). 
bioRxiv preprint doi: https://doi.org/10.1101/2020.09.18.304154; this version posted September 20, 2020. The copyright holder for this preprint (which was not certified by peer review) is the author/funder, who has granted bioRxiv a license to display the preprint in perpetuity. It is made available under aCC-BY-ND 4.0 International license.
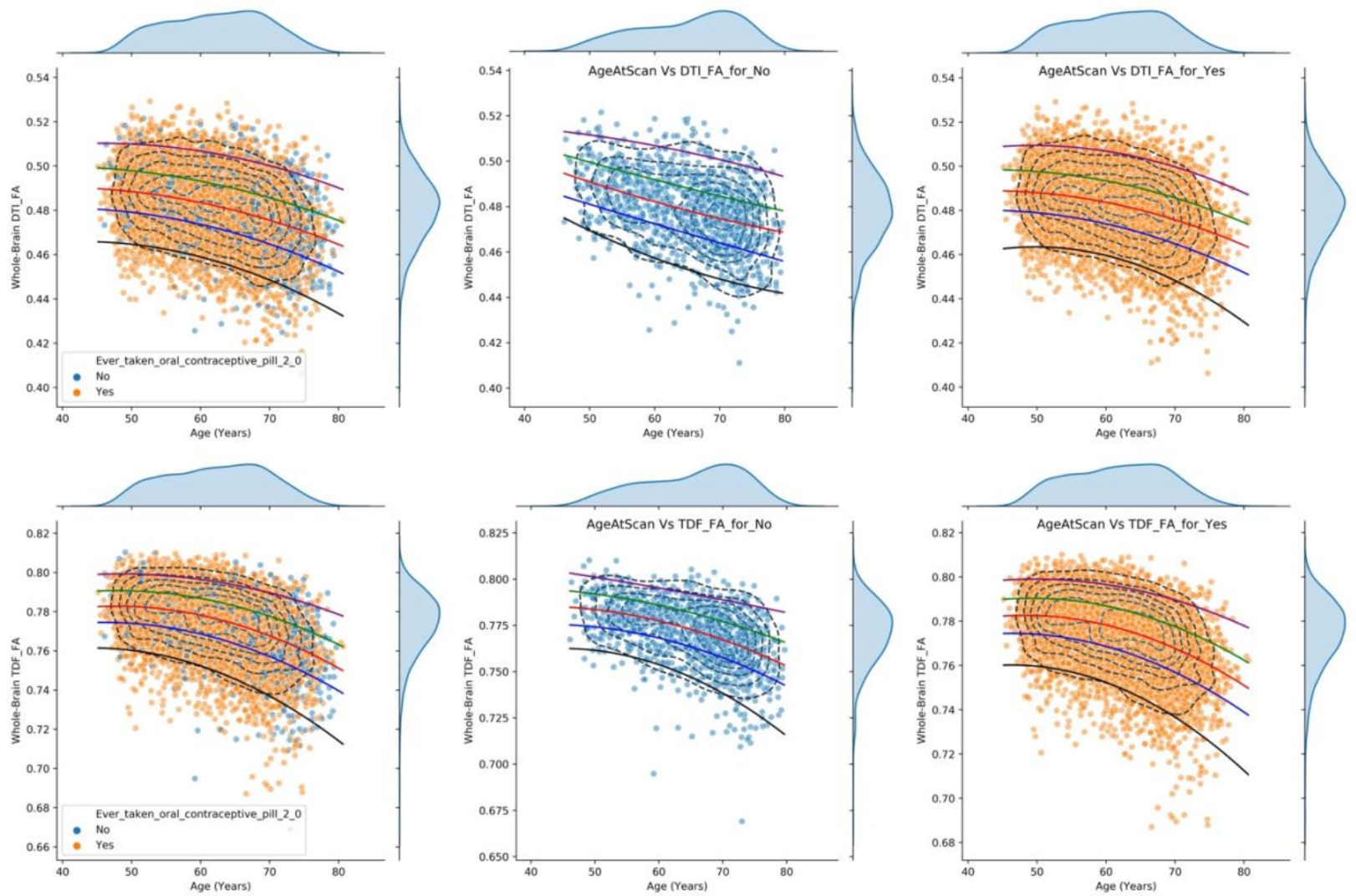

Figure 4. Whole brain averages for DTI-FA (top row) and TDF-FA (bottom row) shown for OC users compared to never-users, collectively (left column) and separately by group (middle and right columns). OC users show slightly higher values of FA (both DTI and TDF) compared to never-users. 


\section{DISCUSSION}

Our results may support the hypothesis that exogenous sex-hormone treatment (HT and OC) has an effect on aging white matter trajectories. It is consistent with our observations that estrogen therapy alone exerts a neuroprotective effect on agerelated white matter processes, compared to hormone replacement therapy containing both estrogen and progestin therapy - which was found to accelerate aging-related processes in women. To our knowledge, we are the first to report these sex hormone effects on white matter aging trajectories, in a large population-based cohort of middle- and older-aged women. We also found that duration of therapy and age of onset show statistical effects on white matter trajectories in women who have used both hormone therapy and oral contraceptive.

Our study was statistically well-powered to detect age associations with white matter microstructure. Even so, the study design has some limitations. Unlike a randomized clinical trial, in which treatments are randomized to individuals, and the effects are studied longitudinally, the current design is a naturalistic study of people using medications. As such, the decision to use treatments, or even have access to these treatments, may in turn depend on health factors not modeled in this study. As such, we report associations that may still be important in understanding brain aging trends in a broad and inclusive population, and suggest mechanisms that could be tested in a causally informed design (such as an RCT). We also note that the term "trajectories" may differ when inferred from cross-sectional rather than longitudinal data, as individuals at different parts of the age range may differ from each other in their childhood developmental experiences, environments, and health risks throughout life. Also, attrition of individuals late in life (due to mortality, or ill health) means that older individuals who participate in the study may show less steep aging trajectories than a typical individual in the population (selection bias, or survivor bias).

Our results show statistical effects on white matter microstructural organization (FA), a loss of fiber coherence (ODI) and increased free water (ISOVF) with age in women following sex hormone treatment. A loss of fiber coherence was seen in both HT users and never-users; however, HT users showed a less steep aging trajectory (less pronounced white matter changes with age) than HT never-users, suggesting a protective effect of sex hormones on normal development and decline of white matter in later life. However, these findings should be interpreted carefully as they were not detected using the fractional polynomials approach, which we might expect to fit the data better than traditional linear regression models. This highlights the need to evaluate alternative models when disentangling age-related effects in large datasets such as this.

A closer examination of differences in hormone therapy formulation, in a subset of subjects, revealed a differential effect of estrogen and combination (estrogen and progestin) hormone therapy on aging white matter trajectories in women. These effects were also detected using the fractional polynomial model. Here, estrogen users displayed more pronounced white matter changes (lower microstructural organization and higher free water) compared to combination users.

Taking into account differences in hormone therapy formulation suggest that in our sample not all HT formulations may have a neuroprotective effect. Hormone replacement therapy containing just estrogen may be marginally more beneficial (less pronounced white matter changes) than estrogen plus progestin therapy. Further, the duration of hormone therapy and the age at hormone therapy onset - influenced FA and the dispersion of intra-voxel freely diffusing water molecules with age, whereby in women on estrogen and progestin therapy, older age of HT onset was associated with greater reductions in age-dependent metrics of white matter microstructure. A clinical trial in 4,532 participants (WHIMS data ${ }^{4}$ ), reported that the risk of probable dementia for women taking estrogen plus progestin was around twice that of women who were assigned to the placebo group; evidence of increased risk appeared as early as 1 year after randomization. Other hormone-related risks included stroke. Observing the effects of estrogen plus progestin on dementia within such a short interval could have implications for understanding hormone-related dementia pathogenesis. Substantial evidence suggests that HT formulation, administration, dosage, compound composition, and mode of administration are clinically relevant ${ }^{3,19}$. Thus, based on this evidence and on our findings, compound composition may modulate aging trajectories in women. Future studies should account for such differences to disentangle effects of hormone therapy treatment regime on white matter microstructural decline in women.

There are two main theories regarding hormone therapy effects. The 'healthy cell bias of estrogen action hypothesis' ${ }^{55}$ suggests that neuronal viability and general health - before starting therapy - might be of importance for estrogen to exert 
therapeutic effects; the second theory is known as the 'critical period hypothesis ${ }^{56}$, whereby HT may be neuroprotective if initiated soon after cessation of ovarian function $\left(<5\right.$ years from menopause $\left.{ }^{16,17}\right)$. The first hypothesis may be relevant for women undergoing surgeries that can cause a drop in circulating estrogen levels such as hysterectomy and/or oophorectomy; however, a history of hysterectomy and/or oophorectomy was accounted for in the present study, and this did not alter findings. Our results support the 'critical period hypothesis' for estrogens, as an earlier age at HT (estrogen only) initiation was associated with less evident white matter aging (particularly before menopause, as findings did not change when covarying for age at menopause).

A reduction in microstructural organization (FA) was seen in birth control users and never-users; however, birth control users showed a slightly less steep age trajectory than never-users, suggesting a protective effect of birth control over white matter aging trajectories. These findings were detected using both traditional regression and fractional polynomial approaches. Prior investigations in the UK Biobank reported reductions in cortical gray matter volume in OC users relative to never-users, worsening by duration of OC treatment ${ }^{25}$. Although longitudinal studies are needed to draw conclusions, it is possible that chronic ovarian hormone suppression (induced by OC treatment) may progressively affect white matter microstructure (as well as cortical volume) later in life. However, we cannot exclude that the direction of any impact of OC on the brain's white matter depends on the type of OC formulation, which was not available in the current study. It was not possible to investigate age associations and birth control formulations with the currently available data in the UK Biobank. As birth control formulations vary from ehinylestradiol-only formulations to those with added concentrations of progestin, we cannot exclude that differences in formulation might exert a differential effect on diffusion metrics ${ }^{3,18}$, so future studies should disentangle effects of birth control formulation on aging trajectories in women.

Besides using multiple statistical approaches, a strength of the current work was the inclusion of multiple diffusion metrics, from tensor-based to "beyond-tensor" models to provide a richer understanding of the underlying white matter structure than traditional diffusion metrics alone. Besides the tensor-based metric FA, we considered the estimated number of crossing fibers, fiber complexity, neurite dispersion and estimated numbers of fiber compartments. FA can lack specificity as it does not directly delineate changes in tissue microstructure - for example a reduction in FA can be associated with different types of microstructural changes, such as demyelination, or a reduction in axonal density. A decline in FA with age could result from many cellular factors, including decreases in the density or an increase in the dispersion orientation of neurites ${ }^{57}$. Multi-compartment models such as NODDI attempt to overcome this by modeling diffusion data using a set of indices that are more directly related to white matter microstructure. In this study, NODDI metrics affected by sex hormone therapy included ODI and ISOVF. ODI quantifies the coherence of fiber orientation: lower values indicate more aligned fibers - here suggesting increased axonal dispersion (reduced fibers alignment) with age following sex hormone therapy. ISOVF represents the fraction of water molecules that are not restricted or directed - an increase that may be consistent with increased dispersion of fibers (ODI) following HT therapy. Free water may also be an index of neuroinflammation as neuroinflammatory processes can lead to increases in the fractional volume of water molecules diffusing freely in the extracellular space.

TBSS is a widely used method for whole-brain voxel-based analysis, but has some limitations. TBSS reduces individual white matter tracts to a skeleton, delineating the center of the tracts and projecting onto it only the highest value of FA along the projection. This may result in loss of microstructural information and potential artifacts ${ }^{58}$, some due to misregistration. In this study, we carried out careful qualitative and quantitative assessments of registration for each subject's diffusion metric. TBSS has limited anatomical specificity (adjacent tracts may not be distinguished) and thus is susceptible to false positives ${ }^{58}$. We may have partially alleviated this problem by fitting increasingly complex diffusion models (DTI $<\mathrm{TDF}<\mathrm{NODDI}$ ). Also, while these limitations may arise for tract-specific diffusion metrics, we chose to focus our analyses on whole-brain diffusion averages.

\section{CONCLUSIONS}

Exogenous sex hormone exposure may impact white matter aging trajectories; white matter metrics may be also be sensitive to sex hormone levels in women. The cross-sectional nature of this study does not enable causal inference, so future studies should use longitudinal designs which are vital to fully understand how estrogen exposure influences brain aging across the lifespan. The various effects of exogenous and endogenous sex hormones on brain function deserve greater critical observation to unravel possible pathogenetic mechanisms, as well as to identify individuals at high risk of 
hormone therapy-related adverse consequences such as dementia or AD. Future studies may benefit from including further clinical and plasma measures and examining genotype interactions (e.g., with apolipoprotein E genotype) to better understand how estrogen exposure influences brain aging and genetic predisposition for neurological disorders in women. Such studies have long-term public health implications and may eventually improve early detection, clinical intervention, and quality of life for individuals at risk for age-related neurodegenerative disorders.

Finally, this study shows the value of testing alternative models for lifespan trajectories beyond popular linear and quadratic models, especially when dealing with large samples. The MFP approach may offer a more flexible alternative to linear or quadratic models, and may be useful for estimating age effects on health outcomes. Large scale datasets such as the UK biobank offer the opportunity to test alternative models for lifespan trajectories beyond traditional statistical models. Studies with a large number of data points may benefit from evaluating findings across alternative explanatory models to understand the robustness of findings.

\section{ACKNOWLEDGMENTS}

This study was supported by NIH grants R01 AG060610, R56 AG058854, U54 EB020403 from the Big Data to Knowledge (BD2K) program, R01 AG059874, P41 EB015922, and a grant from Biogen Inc. (to PMT and NJ).

\section{REFERENCES}

[1] Jones D. K., "Studying connections in the living human brain with diffusion MRI." Cortex 44(8), 936-952 (2008).

[2] Song, Y. J., Li, S. R., Li, X. W., Chen, X., Wei, Z. X., Liu, Q. S., \& Cheng, Y., "The Effect of Estrogen Replacement Therapy on Alzheimer's Disease and Parkinson's Disease in Postmenopausal Women: A Meta-Analysis." Frontiers in Neuroscience, 14, 157 (2020).

[3] Savolainen-Peltonen, H., Rahkola-Soisalo, P., Hoti, F., Vattulainen, P., Gissler, M., Ylikorkala, O., \& Mikkola, T. S., "Use of postmenopausal hormone therapy and risk of Alzheimer's disease in Finland: nationwide case-control study.” BMJ, 364, 1665 (2019).

[4] Shumaker, S. A., Legault, C., Rapp, S. R., Thal, L., Wallace, R. B., Ockene, J. K., ... \& Kotchen, J. M., “Estrogen plus progestin and the incidence of dementia and mild cognitive impairment in postmenopausal women: the Women's Health Initiative Memory Study: a randomized controlled trial." JAMA 289(20), 2651-2662 (2003).

[5] Galea, L. A., Leuner, B., \& Slattery, D. A., "Hippocampal plasticity during the peripartum period: influence of sex steroids, stress and ageing." Journal of neuroendocrinology 26, 641-648 (2014).

[6] Simerly, R. B., "Wired for reproduction: organization and development of sexually dimorphic circuits in the mammalian forebrain." Annual Review of Neuroscience, 25, 507-536 (2002).

[7] Boyle C. P., Raji C. A., Erickson K. I., Lopez O. L., Becker T. J., Gach H. M., Kuller L. H., Longstreth W. T., Carmichael O.T., Thompson P.M., "Estrogen, Brain Structure, and Cognition in Post-Menopausal Women." Hum Brain Mapp, 1- 12 (2020)

[8] Hoekzema, E., Barba-Müller, E., Pozzobon, C., Picado, M., Lucco, F., García-García, D., Soliva, J. C., Tobeña, A., Desco, M., Crone, E. A. et al., "Pregnancy leads to long-lasting changes in human brain structure." Nature Neuroscience, 20, 287 (2017).

[9] Barth, C., Steele, C. J., Mueller, K., Rekkas, V. P., Arélin, K., Pampel, A., Burmann, I., Kratzsch, J., Villringer, A., \& Sacher, J., "In-vivo dynamics of the human hippocampus across the menstrual cycle." Scientific reports, 6, 32833 (2016)

[10] Lisofsky, N., Mårtensson, J., Eckert, A., Lindenberger, U., Gallinat, J., \& Kühn, S., "Hippocampal volume and functional connectivity changes during the female menstrual cycle." NeuroImage 118, 154-162 (2015).

[11] den Heijer, T., Geerlings, M. I., Hofman, A., de Jong, F. H., Launer, L. J., Pols, H. A., \& Breteler, M. M. "Higher estrogen levels are not associated with larger hippocampi and better memory performance." Archives of Neurology 60, 213-220 (2003).

[12] Resnick, S. M., Espeland, M. A., Jaramillo, S. A., Hirsch, C., Stefanick, M. L., Murray, A. M., Ockene, J., Davatzikos, C. et al., "Postmenopausal hormone therapy and regional brain volumes: the whims-mri study." Neurology 72, 135142 (2009). 
[13] Kantarci, K., Tosakulwong, N., Lesnick, T. G., Zuk, S. M., Gunter, J. L., Gleason, C. E., Wharton, W., Dowling, N. M., Vemuri, P., Senjem, M. L. et al., "Effects of hormone therapy on brain structure: a randomized controlled trial." Neurology 87, 887-896 (2016).

[14] Erickson, K. I., Colcombe, S. J., Raz, N., Korol, D. L., Scalf, P., Webb, A., Cohen, N. J., McAuley, E., \& Kramer, A. F. "Selective sparing of brain tissue in postmenopausal women receiving hormone replacement therapy." Neurobiology of Aging 26, 1205-1213 (2005).

[15] Ha, D. M., Xu, J., \& Janowsky, J. S., "Preliminary evidence that long-term estrogen use reduces white matter loss in aging." Neurobiology of aging 28, 1936-1940 (2007).

[16] MacLennan AH, et al., "Hormone therapy, timing of initiation, and cognition in women aged older than 60 years: the remember pilot study." Menopause 13(1):28-36 (2006).

[17] Gibbs RB, Gabor R., "Estrogen and cognition: applying preclinical findings to clinical perspectives." Journal of Neuroscience Research 74(5):637-643 (2003).

[18] Espeland, M. A., Brinton, R. D., Hugenschmidt, C., Manson, J. E., Craft, S., Yaffe, K., ... \& Jackson, R. D, "Impact of type 2 diabetes and postmenopausal hormone therapy on incidence of cognitive impairment in older women." Diabetes Care, 38(12), 2316-2324 (2015).

[19] Gleason, C. E., Dowling, N. M., Wharton, W., Manson, J. E., Miller, V. M., Atwood, C. S., ... \& Neal-Perry, G., "Effects of hormone therapy on cognition and mood in recently postmenopausal women: findings from the randomized, controlled KEEPS-cognitive and affective study." PLoS Med, 12(6), e1001833 (2015).

[20] Hodis, H. N., Mack, W. J., Henderson, V. W., Shoupe, D., Budoff, M. J., Hwang-Levine, J., ... \& Stanczyk, F. Z., "Vascular effects of early versus late postmenopausal treatment with estradiol." NEJM, 374(13), 1221-1231 (2016).

[21] Christin-Maitre, S., "History of oral contraceptive drugs and their use worldwide." Best Practice \& Research Clinical Endocrinology \& Metabolism, 27, 3-12 (2013).

[22] Pletzer, B. A., \& Kerschbaum, H. H., "50 years of hormonal contraception-time to find out, what it does to our brain." Frontiers in Neuroscience 8, 256 (2014).

[23] Mordecai KL, Rubin LH, Maki PM. "Effects of menstrual cycle phase and oral contraceptive use on verbal memory." Hormones and Behavior 54(2):286-293 (2008).

[24] Gogos A. "Natural and synthetic sex hormones: effects on higher-order cognitive function and prepulse inhibition." Biological Psychology 93(1):17-23. (2013).

[25] de Lange, A. M. G., Barth, C., Kaufmann, T., Maximov, I., van der Meer, D., Agartz, I., \& Westlye, L. T. “Cumulative estrogen exposure, APOE genotype, and women's brain aging-a population-based neuroimaging study." bioRxiv, 826123. (2019).

[26] de Lange, A. M. G., Barth, C., Kaufmann, T., Maximov, I. I., van der Meer, D., Agartz, I., \& Westlye, L. T. “Women’s brain aging: effects of sex-hormone exposure, pregnancies, and genetic risk for Alzheimer's disease." bioRxiv, 826123 (2020).

[27] Miller K. L., Alfaro-Almagro F., Bangerter N. K., et al., "Multimodal population brain imaging in the UK Biobank prospective epidemiological study." Nature Neuroscience 19(11), 1523-1536 (2016).

[28] Alfaro-Almagro F., Jenkinson M., Bangerter N. K., et al., "Image processing and Quality Control for the first 10,000 brain imaging datasets from UK Biobank." NeuroImage 166, 400-424 (2018).

[29] Veraart J, et al. "Denoising of diffusion MRI using random matrix theory." NeuroImage 142:394-406 (2016).

[30] Veraart J, Fieremans E, Novikov DS. "Diffusion MRI noise mapping using random matrix theory." Magnetic Resonance in Medicine 76(5):1582-1593 (2016).

[31] Kellner E, Dhital B, Kiselev VG, Reisert M., "Gibbs-ringing artifact removal based on local subvoxel-shifts." Magnetic Resonance in Medicine 76(5):1574-1581 (2016).

[32] Andersson JL, Sotiropoulos SN. "An integrated approach to correction for off-resonance effects and subject movement in diffusion MR imaging." NeuroImage 125:1063- 1078 (2016).

[33] Andersson JL, Graham MS, Zsoldos E, Sotiropoulos SN. "Incorporating outlier detection and replacement into a nonparametric framework for movement and distortion correction of diffusion MR images." NeuroImage 141:556-572 (2016)

[34] Jones, D. K., \& Cercignani, M., "Twenty-five pitfalls in the analysis of diffusion MRI data." NMR in Biomedicine, 23(7), 803-820 (2010).

[35] Pierpaoli, C., Jezzard, P., Basser, P. J., Barnett, A., \& Di Chiro, G., "Diffusion tensor MR imaging of the human brain.” Radiology, 201(3), 637-648 (1996).

[36] Leow A. D., Zhu S., Zhan L., et al., "The tensor distribution function.” Magnetic Resonance in Medicine 61(1), 205214 (2009). 
[37] Nir T. M., Jahanshad N., Villalon-Reina J. E., et al., "Fractional anisotropy derived from the diffusion tensor distribution function boosts power to detect Alzheimer's disease deficits." Magnetic Resonance in Medicine 78(6), 2322-2333 (2017).

[38] Villalon-Reina, J. E., Ching, C. R., Kothapalli, D., Sun, D., Nir, T., Lin, A., ... \& Hansen, L. “Alternative diffusion anisotropy measures for the investigation of white matter alterations in 22q11.2 deletion syndrome." Proc SPIE 10975, $1-13$ (2018).

[39] Zhang H., Schneider T., Wheeler-Kingshott C. A. and Alexander D. C., "NODDI: practical in vivo neurite orientation dispersion and density imaging of the human brain," Neuroimage 61(4), 1000-1016 (2012).

[40] Daducci, A., Canales-Rodríguez, E. J., Zhang, H., Dyrby, T. B., Alexander, D. C., \& Thiran, J. P., "Accelerated microstructure imaging via convex optimization (AMICO) from diffusion MRI data." NeuroImage 105, 32-44 (2015).

[41] Smith, S. M., Jenkinson, M., Johansen-Berg, H., Rueckert, D., Nichols, T. E., Mackay, C. E., ... \& Behrens, T. E. "Tract-based spatial statistics: voxelwise analysis of multi-subject diffusion data." NeuroImage 31(4), 1487-1505 (2006).

[42] Mori, S., Wakana, S., Van Zijl, P. C., \& Nagae-Poetscher, L. M, "MRI atlas of human white matter." Elsevier (2005).

[43] Wakana, S., Caprihan, A., Panzenboeck, M. M., Fallon, J. H., Perry, M., Gollub, R. L., ... \& Blitz, A. "Reproducibility of quantitative tractography methods applied to cerebral white matter." NeuroImage 36(3), 630-644 (2007).

[44] Royston P, Altman DG, "Regression using fractional polynomials of continuous covariates: parsimonious parametric modelling." Appl Stat 43:429-467 (1994).

[45] Sauerbrei W., Meier-Hirmer C., Benner A., Royston P., "Multivariable regression model building by using fractional polynomials: description of SAS, STATA and R programs." Comput Stat Data An 50: 3464-3485 (2006).

[46] Nobis L., Manohar S. G., Smith S. M., et al., "Hippocampal volume across age: Nomograms derived from over 19,700 people in UK Biobank." NeuroImage Clin 23, 101904 (2019).

[47] Bethlehem R. A. I., Seidlitz J., Romero-Garcia R., Dumas G. and Lombardo M. V., "Normative age modelling of cortical thickness in autistic males." bioRxiv, 1-23 (2019).

[48] Lv J., Di Biase M., Cash R. F. H., et al., "Individual deviations from normative models of brain structure in a large cross-sectional schizophrenia cohort." bioRxiv 911032, (2020).

[49] Goujon A, Samir KC, Speringer M, Barakat B, Potancoková M, Eder J, Striessnig E, Bauer R, Lutz W. “A harmonized dataset on global educational attainment between 1970 and 2060- An analytical window into recent trends and future prospects in human capital development." J Demogr Econ, 82(3):315-63 (2016).

[50] Gadekar T, Dudeja P, Basu I, Vashisht S, Mukherji S., "Correlation of visceral body fat with waist-hip ratio, waist circumference and body mass index in healthy adults: A cross sectional study." Medical Journal Armed Forces India. (2018).

[51] Lee HJ, Hwang SY, Hong HC, Ryu JY, Seo JA, Kim SG, Kim NH, Choi DS, Baik SH, Choi KM, Yoo HJ., "Waistto-hip ratio is better at predicting subclinical atherosclerosis than body mass index and waist circumference in postmenopausal women." Maturitas. 80:323-328 (2015).

[52] Scicali R, Rosenbaum D, Di Pino A, Giral P, Cluzel P, Redheuil A, Piro S, Rabuazzo AM, Purrello F, Bruckert E, Gallo A. "An increased waist-to-hip ratio is a key determinant of atherosclerotic burden in overweight subjects." Acta Diabetol. 55:741-749 (2015).

[53] Townsend P, Phillimore P, Beattie A., "Health and deprivation: inequality and the North." Routledge (1988).

[54] Smith GD, Whitley E, Dorling D, Gunnell D., "Area based measures of social and economic circumstances: cause specific mortality patterns depend on the choice of index." J Epidemiol Community Health 55:149-150 (2001).

[55] Brinton R. D., "The healthy cell bias of estrogen action: mitochondrial bioenergetics and neurological implications." Trends in Neurosciences 31(10):529-537 (2008).

[56] Maki P.M., "The critical window hypothesis of hormone therapy and cognition: a scientific update on clinical studies." Menopause (New York, NY) 20(6):695 (2013).

[57] Pines, A. R., Cieslak, M., Larsen, B., Baum, G. L., Cook, P. A., Adebimpe, A., ... \& Oathes, D. J., "Leveraging multishell diffusion for studies of brain development in youth and young adulthood." Developmental Cognitive Neuroscience, 100788 (2020).

[58] Bach, M., Laun, F. B., Leemans, A., Tax, C. M., Biessels, G. J., Stieltjes, B., \& Maier-Hein, K. H., "Methodological considerations on tract-based spatial statistics (TBSS).” NeuroImage, 100, 358-369 (2014). 